\title{
Characteristic Gait Animation Synthesis from Single View Silhouette
}

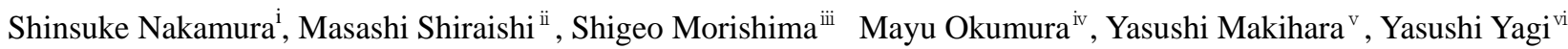 \\ Waseda University \\ Osaka University
}

\section{Introduction}

Characteristics of human motion, such as walking, running or jumping vary from person to person. Differences in human motion enable people to identify oneself or a friend. However, it is challenging to generate animation where individual characters exhibit characteristic motion using computer graphics. Our goal is to construct a system that synthesizes characteristic gait animation automatically. As a result, when crowd animation is generated for instance, the motion with the variation can be made using our system. In our system, we first acquire a silhouette image as input data using a video camera. Second, we extract gait feature from single view silhouette. Finally we automatically synthesize 3D gait animation using the method blending a small number of motion data [KOVAR, L et al 2003].This blending weight is estimated using the gait feature automatically.

\section{Extraction Gait Feature}

We record a walker who goes straight from side view using a video camera. For the purpose of extracting gait silhouette from background easily, we set up the background of single color.

The height of silhouette image acquired from video camera is normalized, because we don't consider the size of body.

In this paper we extract the width of step, the area where arm sweep, and the degree of stoop as gait feature $g_{j} \cdot j=1,2,3$ from silhouette image.

\section{Estimation Blending Weight}

First of all, we must prepare a small number of motions for synthesizing new motion beforehand. For our experiment, a participant was required to walk on a treadmill at a speed of $4[\mathrm{~km} / \mathrm{h}]$, while we acquired 3D motion data using Mocap. The motion data is data of $i=1,2, \ldots 6$ kinds of walking styles, such as large or small width of step, large or small area where arm sweep, stoop, and recurved, which are cut out of two steps from the left leg landing. These motions are represented by $M_{i}\left(f_{i}\right)$, and called key motions. $f_{i}$ is number of frame. We presented the method of estimating blending weight $w_{i}$ First, we extract the gait features $g_{j}\left(M_{i}\right)$ using the $3 \mathrm{DCG}$ character animation made of the key motions $M_{i}$. The gait feature of synthesized motion $\hat{g}$ is represented (1)

$$
\hat{g}=w_{i} g\left(M_{i}\right)
$$

Input data $g$ is extracted using the silhouette image by recording a walker. Second, we estimate blending weight $w_{i}$ that minimizing Euclidean distance between $g$ and $\hat{g}$ is minimized using the active set method of convex quadratic programming problem.

$$
\begin{aligned}
& D=\|\hat{g}-g\|^{2} \rightarrow \min \\
& \sum_{i} w_{i}=1 \\
& 0 \leq w_{i} \leq 1
\end{aligned}
$$

Finally, we recalculate blending weight $w_{i}$ by weighting to the gait feature which is the nearest input data $g$ in the gait features $g_{j}\left(M_{i}\right)$.

\section{Models for gait animation synthesis}

As for key motions $M_{1}, \ldots M_{i}$, the frame $f_{i}$ and timing are different. Linear blending of each frame cannot be done using $M_{1}, \ldots M_{i}$. We synchronize all the motions using the method of time warping [KOVAR, L et al 2003]. Synthesized motion $\hat{M}$ is by linear blending synchronized motions $S_{1} \ldots S_{i}$.

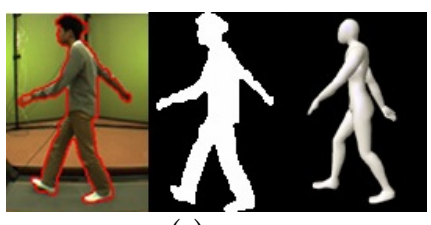

(a)

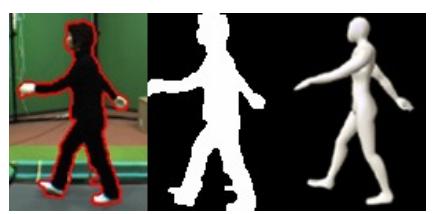

(c)

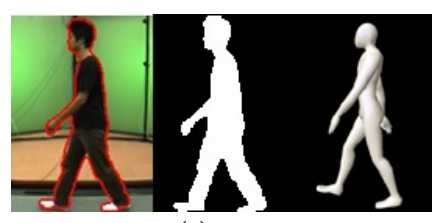

(b)

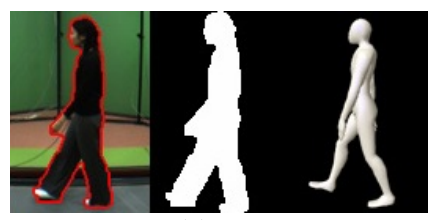

(d)
Figure 1: Example of input data, silhouette, and synthesized gait animation

The blending weight $w_{i}$ is the value estimated from the gait feature.

$$
\hat{M}=\sum w_{i} S_{i}
$$

The key motions $M_{1}, \ldots M_{i}$ are the same all speeds, and frame $f_{i}$ is different according to the width of step.

Therefore, the frame of synthesized motion should be determined by its step. The relation between the frame and the step of key motions $M_{1}, \ldots M_{i}$ is applied to the expression linear approximated, and the frame of the synthesized motion is calculated by its step. The Synthesized motion is expanded to the size of the calculated frame using the method of time warping. If we use this motion as long walking animation, we smooth this motion in order to keep continuousness.

\section{Results}

We show synthesized gait animation using our system at Fig 1. For example, the gait feature extracted from the participant shown (a) is a large degree of stoop, and (c) is a large area where arm sweep. These synthesized gait animation can express the feature of walking enough. Very delicate feature like small difference walking (b) and walking (d) in Fig.1 can be expressed in our system.

\section{Future Work}

In this paper we presented the method of constructing a system that synthesizes characteristic gait animation automatically from gait feature using single view silhouette. As future work, we need to increase the number of walking styles and a number of gait features for representing all walkers in the world. In this paper model of body is only one. However, each person's body is different size and proportion. Therefore, we should synthesize animation including not only information of motion, but also body. Finally, it is necessary to evaluate how accuracy original walker and synthesized animation look like.

\section{References}

Y. Makihara, R. Sagawa, Y. Mukaigawa, T. Echigo, Y. Yagi, "Gait Recognition Using a View Transformation Model in the Frequency domain", Proc.of the $9^{\text {th }}$ European Conf.on Computer Vision (ECCV2006), Vol.3, pp.96-99, 2006 L,Kovar, M, Gleicher, "Flexible Automatic Motion Blending with Registration Curves", SIGGRAPH2003, pp.214-224, 2003

\footnotetext{
i e-mail: pencil@moegi.waseda.jp

ii e-mail: s-masashi331@moegi.waseda.jp

iii e-mail: shigeo@waseda.jp

iv, v, vi e-mailः \{okumura, makihara, yagi\}@am.sanken.osaka-u.ac.jp
} 